\title{
Link, Twist, Energy, and the Stability of DNA Minicircles
}

\author{
Kathleen A. Hoffman ${ }^{1}$, Robert S. Manning ${ }^{2}$, \& John H. Maddocks ${ }^{3}$ \\ khoffman@math.umbc.edu, rmanning@haverford.edu, john.maddocks@epfl.ch
}

November 15, 2002

Submitted to Biopolymers.

We describe how the stability properties of DNA minicircles can be directly read from plots of various biologically intuitive quantities along families of equilibrium configurations. Our conclusions follow from extensions of the mathematical theory of distinguished bifurcation diagrams $[9,21]$ that are applied within the specific context of an elastic rod model of minicircles. Families of equilibria arise as a twisting angle $\alpha$ is varied. This angle is intimately related to the continuously varying linking number $L k$ for nicked DNA configurations that is defined as the sum of Twist and Writhe. We present several examples of such distinguished bifurcation diagrams involving plots of the energy $E$, linking number $L k$, and a twist moment $m_{3}$, along families of cyclized equilibria of both intrinsically straight, and intrinsically curved, DNA fragments.

\footnotetext{
${ }^{1}$ Department of Mathematics and Statistics; University of Maryland, Baltimore County; Baltimore, MD 21250 USA

${ }^{2}$ Mathematics Department; Haverford College; Haverford, PA 19041 USA

${ }^{3}$ Bernoulli Institute; École Polytechnique Fédérale de Lausanne; CH-1015 Lausanne Switzerland
} 


\section{Introduction}

DNA cyclization has proven to be a convenient experimental setting for probing the physical behavior of segments of DNA under biologically realistic conditions, see, e.g., [13, 28, 16, 14, 4]. If experiments are performed on sufficiently short DNA (around 200 basepairs (bp), for example) then the experimentally pertinent configurations appear to have approximately circular centerlines, and thus do not involve self-contact, i.e., close interaction of remote parts of the sequence. In this case, the experiments provide a sensitive probe of purely local, sequencedependent, mechanical properties of the DNA, such as intrinsic shape and bending and twisting flexibilities.

An efficient computational model of cyclization is necessary to relate DNA shape and flexibility parameters to the available experimental cyclization data. Short DNA fragments of only a few hundred bp in length are still beyond the capabilities of today's molecular dynamics simulations, so that some sort of coarse-grained approximation must be used in any cyclization model. There are a variety of coarse-grained models available (see, e.g., [27, 25] for reviews), involving, for example, rigid basepair approximations (e.g., [20, 26]), piecewise linear approximations to the DNA centerline (e.g., $[5,15]$ ), or continuum elastic rod models (e.g., [2, 29]). In particular, and reflecting the increasing level of sophistication and detailed information available from experiment, there have been a number of works that consider continuum elastic rod models for DNA with an increasing level of refinement. Within continuum theories, which can all be described as rod models, there is a hierarchy of descriptions evolving from basic and fundamental models with intrinsically straight rods of uniform rigidities and circular cross-section, where analytic solutions can provide useful insights, to models capturing sequence-dependence through natural curvature, non-uniform rigidities, and anisotropic cross-sections, where solutions can only be found numerically.

In prior work we have used the computational approach of parameter continuation within a continuum rod model [23, 7] of DNA minicircles. The primary objective of this article is to describe certain attractive aspects of this computational approach from a specifically biological point of view. Two such features, whose biological pertinence is at first sight perhaps nonintuitive, are a) the computations characterize all equilibrium configurations i.e., not only local minima, but rather any critical (or stationary) point of the DNA mechanical strain energy $E$, minimal or not, and b) the computations include DNA equilibria in which the strands of the DNA double helix are not torsionally aligned at one basepair step. These two special features offer several advantages.

Knowledge of all equilibrium configurations within the model provides a complete skeleton of the potential energy landscape. Of course, it is those equilibria realizing local minima that often make the most significant contributions to experimental observations, and to the Boltzmann distribution of configurations sampled during the thermally driven motion of the DNA, but other types of equilibria are important too. For example the value of the potential energy $E$ at a saddle point plays an important role in fixing the energy barrier between different local minima. Similarly, as a problem parameter is varied, a saddle can split into two saddles and a new local minimum. We remark that only local minima are accessible to computational techniques such as direct numerical minimization or simulated annealing of the energy, whereas parameter 
continuation applied to the equilibrium equations naturally tracks all types of equilibria.

Once an equilibrium has been computed using parameter continuation, it must still be classified as being either a local minimum or a saddle point. We will refer to this as the stability of the critical point or equilibrium: local minima will be called stable and other critical points unstable. More generally, we can assign an index to an equilibrium corresponding to the number of independent directions in configuration space that can decrease the potential energy $E$ : local minima have index 0 , saddles with one downward direction have index 1 , etc. With the appropriate mathematical machinery, it is rather straightforward to determine the index of each equilibrium once it has been embedded in a family of equilibria that have been computed using parameter continuation [24, 8].

The inclusion of torsionally-misaligned configurations within our model is a convenient computational strategy for embedding the true cyclized configurations, which are of primary biological interest, in a family of equilibria. If one attempts to determine only cyclized configurations, then one must know in advance how many there are, and have good initial approximations for each. On the other hand, in our extended model, cyclized equilibria lie on curves of torsionallymisaligned critical points as the angle $\alpha$ of misalignment is varied, and usually several cyclized equilibria lie on each curve. Thus, one modifies the problem of finding many isolated cyclized critical points to the problem of first finding a small number of curves of critical points within the extended torsionally-misaligned problem, and then identifying which points on the curve are actually cyclized. To start the computation one need only know a good approximation to one point on the curve, and such points can readily be computed starting from analytic solutions of simplified models. In practice, only one or two such curves must be computed in order to find all pertinent cyclized local minima, and the entire computation is not costly (generally requiring at most a few minutes on a current workstation).

In mathematical language, within the extended, torsionally-misaligned model, the basic object to be computed is a one-parameter bifurcation diagram, i.e., a set of curves of DNA equilibria as the angle $\alpha$ of misalignment at the nick site is varied. In this article we shall explain how the mathematical theory of stability exchange can be used to read off the change in index of each equilibrium from the shape of certain distinguished plots (along the curve of equilibria) of energy $E$, linking number (or just link) $L k$, and a twist moment $m_{3}$.

Our discussion of $L k$ requires the extension of the definition of link to the torsionallymisaligned case. In the literature of modeling DNA much use has been made of the remarkable formula $L k=T w+W r$, which, in the cyclized case, is a theorem relating the three independently defined quantities link $L k$, a topologically defined integer, and the real numbers twist $T w$ and writhe $W r$. In the torsionally-misaligned case the topological definition of $L k$ is no longer valid, so, following [15], we are free to use the formula $L k=T w+W r$ as the definition of $L k$ (and in general this $L k$ is not an integer). In fact, $L k$ defined in this fashion and the misalignment angle $\alpha$ are intimately related. This relationship is perhaps rather intuitive, and is mentioned without comment in [15], for example. Nevertheless we are unaware of a prior complete argument demonstrating its validity, and, given its importance in our work, we provide in an Appendix a complete proof in terms of a new notion that we call the writhe frame, which is itself perhaps a notion of some independent interest.

While our motivation for introducing torsionally misaligned minicircles is primarily compu- 
tational, it is also true that experiments can be carried out on circular DNA with one nicked strand. We remark that the torsionally misaligned configurations we compute may or may not be good models for such real nicked DNA. In particular, the potential difficulty lies in assigning physical parameters to accurately mimic the properties of the DNA double helix at the nick site. However if models of the nick of this type are accepted, as is done in [15], for example, then, as discussed in [7], our parameter continuation techniques have a final attractive feature: computation of the bifurcation diagram for a family of prescribed angles $\alpha$ can rather accurately predict the equilibrium distribution $P(L k)$ for short nicked minicircles.

\section{Elastic Rod Model}

The Cosserat elastic rod is a standard model in continuum mechanics [1]. We think of a rod as a long slender object, and consider slicing it into a continuous family of cross-sections. With DNA, the basepairs provide a natural choice for these cross-sections (though, of course, some smoothing strategy must be applied in order to convert to a continuum setting [23]). We denote the position of the center of mass of each cross-section by $\mathbf{r}$ and parametrize by arclength to get a center curve $\mathbf{r}(s)$. We describe each cross-section using a frame of three orthonormal vectors $\left(\mathbf{d}_{\mathbf{1}}(s), \mathbf{d}_{\mathbf{2}}(s), \mathbf{d}_{\mathbf{3}}(s)\right)$, with $\mathbf{d}_{\mathbf{3}}$ normal to the plane of the cross-section and $\mathbf{d}_{\mathbf{1}}, \mathbf{d}_{\mathbf{2}}$ in its plane (for example, for DNA, one may choose $\mathbf{d}_{\mathbf{1}}$ to point to the center of the major groove). We refer

to $\left(\mathbf{d}_{\mathbf{1}}, \mathbf{d}_{\mathbf{2}}, \mathbf{d}_{\mathbf{3}}\right)$ as a framing of the curve $\mathbf{r}$, and thus the rod as a whole is modeled as a framed curve $\left(\mathbf{r}, \mathbf{d}_{1}, \mathbf{d}_{2}, \mathbf{d}_{3}\right)$. Following prior work in the area, the computations in this paper assume an additional (and independent) inextensibility-unshearability constraint. This constraint can be written as:

$$
\mathbf{r}^{\prime}=\mathbf{d}_{3},
$$

where, here and throughout, a prime indicates differentiation with respect to $s$. In the context of DNA, the constraint (1) can be interpreted as the assumption that the values of rise, slide, and shift do not change appreciably as the DNA deforms. The accuracy of this assumption can be verified a posteriori. For the minicircles modeled in [23], it is certainly a good approximation. In other circumstances, for example single-molecule experiments with a high applied force loadings, the approximation is less good, in which case there are more intricate extensible and shearable rod models which could be considered.

The rod potential energy is naturally expressed in terms of strains $u_{i}\left(u_{1}\right.$ and $u_{2}$ are strains for bending about $\mathbf{d}_{\mathbf{1}}, \mathbf{d}_{\mathbf{2}}$, and $u_{3}$ the strain for twisting about $\mathbf{d}_{\mathbf{3}}$ ):

$$
\begin{aligned}
& u_{1}\left(\mathbf{d}_{1}, \mathbf{d}_{2}, \mathbf{d}_{3}\right) \equiv \mathbf{d}_{3} \cdot \mathbf{d}_{2}^{\prime}=-\mathbf{d}_{2} \cdot \mathbf{d}_{3}^{\prime}, \\
& u_{2}\left(\mathbf{d}_{1}, \mathbf{d}_{2}, \mathbf{d}_{3}\right) \equiv \mathbf{d}_{1} \cdot \mathbf{d}_{3}^{\prime}=-\mathbf{d}_{3} \cdot \mathbf{d}_{1}^{\prime}, \\
& u_{3}\left(\mathbf{d}_{1}, \mathbf{d}_{2}, \mathbf{d}_{3}\right) \equiv \mathbf{d}_{2} \cdot \mathbf{d}_{1}^{\prime}=-\mathbf{d}_{1} \cdot \mathbf{d}_{2}^{\prime} .
\end{aligned}
$$

In the context of DNA, these strains are the continuum analogue of tilt, roll, and twist angles. The energy is then given as an integral over the arclength of the rod:

$$
E=\int_{0}^{L} W\left(u_{1}-\hat{u}_{1}, u_{2}-\hat{u}_{2}, u_{3}-\hat{u}_{3} ; s\right) d s,
$$


where the typical form chosen for $W$ is

$$
W=\frac{1}{2}\left(K_{1}\left(u_{1}-\hat{u}_{1}\right)^{2}+K_{2}\left(u_{2}-\hat{u}_{2}\right)^{2}+K_{3}\left(u_{3}-\hat{u}_{3}\right)^{2}\right) .
$$

The function $W$ gives the local elastic strain energy density of the configuration due to bending and twisting, and integration over the length $L$ gives the total potential energy of the DNA. The form of the function $W$ contains sequence-dependent parameters modeling the DNA minimumenergy shape and flexibility that are assumed within the model. For example, in the particular form $(4), K_{i}(s)$ are bending $(i=1,2)$ and twisting $(i=3)$ stiffnesses and $\hat{u}_{i}(s)$ determine the intrinsic shape of the DNA (e.g., $\hat{u}_{1}=\hat{u}_{2}=0$ implies an intrinsically straight rod). The accuracy of the model certainly depends upon the accuracy to which the parameters appearing in the energy are known, or indeed whether a more general functional form, e.g., one containing non-diagonal cross-terms in (4) or an extensible-shearable model, is more appropriate. However, the general observations that we shall make are independent of the precise form of the energy (3).

One final quantity will be important in our theory, namely the torque or twist moment which we denote $m_{3}$. It is defined by

$$
m_{3}=\frac{\partial W}{\partial u_{3}}
$$

or in the particular case of quadratic energy (4),

$$
m_{3}=K_{3}\left(u_{3}-\hat{u}_{3}\right) .
$$

(There are two additional bending moments defined analogously, but they will not play a prominent role here).

To model cyclization, we seek equilibrium points of the energy $E$ among configurations $\left(\mathbf{r}, \mathbf{d}_{1}, \mathbf{d}_{2}, \mathbf{d}_{3}\right)$ satisfying the inextensibility-unshearability constraint (1), the orthonormality of the $\mathbf{d}_{i}$, and in addition the cyclization boundary conditions (see Fig. 1):

$$
\begin{aligned}
\mathbf{r}(L) & =\mathbf{r}(0) \\
\mathbf{d}_{3}(L) & =\mathbf{d}_{3}(0) \\
\mathbf{d}_{1}(L) & =\cos \alpha \mathbf{d}_{1}(0)+\sin \alpha \mathbf{d}_{2}(0) \\
\mathbf{d}_{2}(L) & =-\sin \alpha \mathbf{d}_{1}(0)+\cos \alpha \mathbf{d}_{2}(0) .
\end{aligned}
$$

Here $\alpha$ is the imposed twist angle at $s=L$ relative to $s=0$. Of course, this angle is only defined modulo $2 \pi$. As we shall discuss later, configurations with $\alpha$ differing by $2 \pi n$ can be interpreted as configurations with different prescribed link $L k$. For convenience, we denote by $\alpha_{0}$ the value for this angle that lies in $[0,2 \pi)$, such that

$$
\alpha=\alpha_{0}+2 m \pi
$$

for some integer $m$. For cyclized DNA, $\alpha_{0}=0$. 


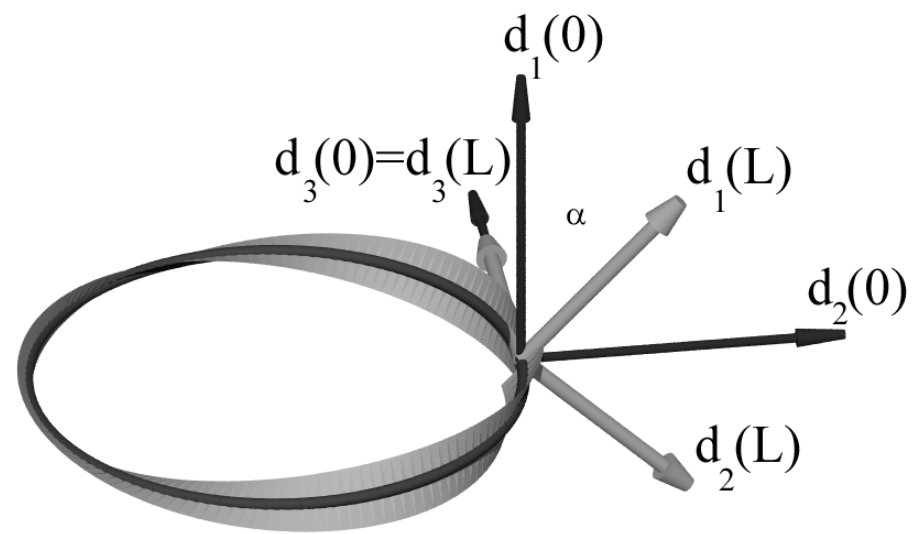

Figure 1: Cyclization constraints on an elastic rod 


\section{The connection between $\alpha$ and $L k$}

The twist angle $\alpha$ is a central parameter in our model, which we vary in computing DNA equilibria. On the other hand, in modeling DNA cyclization, attention is often focused on the link $L k$ of the two sugar-phosphate chains. The two quantities $\alpha$ and $L k$ are intimately related.

In our rod model for cyclized DNA, the curve $\mathbf{r}(s)$ is defined to run through the center of the DNA, and the vector $\mathbf{d}_{1}(s)$ tracks the twist of the sugar-phosphate chains. Because of these definitions, we can continuously deform one of the sugar-phosphate chains, without intersecting itself or the other chain, to lie along $\mathbf{r}(s)$, and then deform the other chain, again without intersecting itself or the first chain, to lie along $\mathbf{r}(s)+h \mathbf{d}_{1}(s)$, for some small radius $h$. Since the link is a topological invariant, it remains constant during these deformations, so that $L k$ equals the link of the curve $\mathbf{r}(s)$ with the curve $\mathbf{r}(s)+h \mathbf{d}_{1}(s)$. In this special setting, we can compute $L k$ via the Călugăreanu-White-Fuller formula $L k=T w+W r[3,31,6]$, in terms of the twist and writhe

$$
\begin{aligned}
T w & =\frac{1}{2 \pi} \int_{0}^{L} u_{3}(s) d s \\
W r & =\frac{1}{4 \pi} \int_{0}^{L} \int_{0}^{L} \frac{(\mathbf{r}(s)-\mathbf{r}(\sigma)) \cdot\left(\mathbf{r}^{\prime}(s) \times \mathbf{r}^{\prime}(\sigma)\right)}{|\mathbf{r}(s)-\mathbf{r}(\sigma)|^{3}} d \sigma d s .
\end{aligned}
$$

When the DNA is torsionally-misaligned, the topological definition of $L k$ no longer holds, as the sugar-phosphate chains are not closed curves. On the other hand, the integrals for $T w$ and $W r$ still exist, so one may define $L k$ to be $T w+W r$. This choice of definition for $L k$ is particularly efficacious, in that it yields a strong connection to the angle $\alpha$, as noted without proof in [15]:

$$
L k=\frac{\alpha}{2 \pi}(\bmod 1) .
$$

In Appendix A, we offer a proof of a slightly stronger result:

$$
L k=m+\frac{\alpha_{0}}{2 \pi}
$$

where $m$ is the link of the nearest undertwisted cyclized DNA and $\alpha_{0}$ is defined by (8). The central idea of the proof is to associate to $\mathbf{r}$ a new writhe frame $\left(\mathbf{d}_{\mathbf{1}}^{\mathbf{w}}, \mathbf{d}_{\mathbf{2}}^{\mathbf{w}}, \mathbf{d}_{\mathbf{3}}\right)$.

The link $L k$ has the advantage that it is uniquely defined for a given configuration, whereas $\alpha$ is only defined modulo $2 \pi$ (although in a given parameter continuation computation, the choice of $\alpha$ is unambiguous; the computation will simply choose the value closest to the value of $\alpha$ in the previous step). On the other hand, $L k$ has the property that whenever the rod centerline passes through itself, the value of $L k$ jumps by 2 . Jumps in $L k$ arise exactly when the model neglecting self-contact is not appropriate.

\section{Families of Equilibria and Bifurcation Diagrams}

In general, the equilibria of cyclized DNA cannot be found analytically. However, there is one simple case in which equilibria are known in closed form: when $\hat{u}_{1}(s) \equiv \hat{u}_{2}(s) \equiv 0$ and 
$K_{1}(s) \equiv K_{2}(s) \equiv A$, uniformly twisted circles are equilibria. These twisted circles can be used as a starting point for the numerical computation of equilibria for intrinsically curved DNA $\left(\hat{u}_{1}, \hat{u}_{2} \neq 0\right)$. Given one such equilibrium for an intrinsically curved DNA, one can find many other (mainly torsionally-misaligned, i.e., $\alpha_{0} \neq 0$ ) equilibria by performing a numerical continuation in the angle $\alpha$. The multivaluedness of $\alpha$ expressed in (8) is crucial here, as we can choose the integer $m$ in such a way that $\alpha$ is continuous throughout a calculation. These computations are not entirely straightforward, but they are discussed at length in [7, 22, 23].

In this article, our main interest lies in understanding the information that can be extracted from computations of a family of equilibria as the angle parameter $\alpha$ is varied. Plots of such a family are called, in the language of mathematics, bifurcation diagrams. In this article, we will focus on the biologically familiar quantities $E$ and $L k$, and the additional quantity $m_{3}$ (at the point of ring closure $s=0, L)$. As $\alpha$ varies, each quantity can be evaluated, and they can be plotted in pairs, such as $\left(m_{3}(\alpha), E(\alpha)\right)$.

We begin with the diagrams for an intrinsically straight and untwisted rod with constant and equal bending stiffnesses $K_{1}(s)=K_{2}(s)=A$, and constant twisting stiffness $K_{3}(s)=C$; this case is sometimes called the perfect rod. The only remaining free parameter is the ratio of stiffnesses $C / A$. In Fig. 2 we show the $\left(m_{3}, L k\right),(E, L k)$, and $\left(E, m_{3}\right)$ diagrams for the perfect rod, for several values of $C / A$. The Figure shows only the lowest-energy piece of the entire diagram, which extends to arbitrarily high energies.

The diagram contains a central "trivial" branch, which is a diagonal line in the $\left(m_{3}, L k\right)$ diagram and a parabola in the $(E, L k)$ and $\left(E, m_{3}\right)$ diagrams. Each equilibrium on this branch is circular with a constant twist. The simplest such equilibrium is the untwisted circle, which occurs at $L k=m_{3}=0$ and $E=2 \pi^{2} A / L$, and is stable. As twist is introduced, i.e., $|L k|$ is increased on this branch, equilibria remain stable until the well-known "first buckling transition", which occurs at the dots in the Figure, with $m_{3}= \pm 2 \pi \sqrt{3} A / L, L k= \pm A \sqrt{3} / C$, and $E=2 \pi^{2} A / L+6 \pi^{2} A^{2} /(C L)[18]$. Thereafter, equilibria on the trivial branch are unstable, with index two initially, and increasing by two at each successive buckling transition (later buckling transitions not pictured in the Figure). At the buckling transition, a second branch of "nontrivial" equilibria emerges, and in fact connects the two first-buckling-transitions. Solutions on this branch are nonplanar, buckling through a figure-eight (at $m_{3}=0$ ). Note that the current model includes no self-contact force, so the branch continues through the figure-eight to equilibria with $L k$ and $m_{3}$ of the opposite sign, and with $L k$ jumping by two. The stability of equilibria on the nontrivial branch depends on $C / A$, as indicated in the Figure. For sufficiently low $C / A$, the entire branch is unstable (of index 1 ). Hoffman has shown that the transition is at $C / A=1.375$ [9]; for $C / A$ above this value, equilibria near the buckling transition are stable, as in the case $C / A=1.8$ shown in the Figure. For $C / A>2.0$, the entire nontrivial branch is stable $[9,12]$.

Next, in Fig. 3, we show diagrams for the cyclization of a 155-bp DNA consisting of an Atract and a CAP binding site connected by several nearly straight DNA segments (the molecule used is 08T15 from [23], and the procedure for deriving continuous DNA shape parameters $\hat{u}_{i}(s)$ from sequence-dependent bend and twist "wedge angles" is described therein). The intrinsic curvature of the A-tracts and, to a lesser extent, the CAP binding site causes the diagrams to perturb from Fig. 2; for example, the energies drop since intrinsic curvature reduces the strain 
energy required to cyclize.

\section{Stability Exchange and Distinguished Bifurcation Di- agrams}

Two features of a bifurcation diagram - folds and bifurcation points - play a central role in predicting the change of stability of equilibria along the diagram. A fold is a point on a branch where one (or more) of the plotted coordinates passes through a local maximum or minimum, such as the points labeled I and IV in column a of Figs. 2 and 3. If both coordinates pass through local extrema at a point, we call the point a cusped fold, such as the point labeled II in column b of Fig. 3.

A bifurcation point is a point on the diagram where two branches cross, such as the dots in Fig. 2. Bifurcation points are rather exceptional; in the absence of a symmetry forcing their appearance, they typically do not arise. Alternatively one can say that the case of folds is generic; if a particular system has a bifurcation point, a nearby system with suitably perturbed coefficients in the energy will have only fold points.

It is a standard result in bifurcation theory that stability properties of a branch of solutions can only change at a fold in the continuation parameter $\alpha$, or exceptionally at a bifurcation point $[11,30]$. Maddocks and Hoffman have strengthened this result to predict the exact way the stability index changes at a fold in problems involving equilibria of an energy (such as the DNA minicircle example) [9, 21]. Our objective here is to summarize this general stability exchange theory and then use it to create bifurcation diagrams involving biologically familiar coordinates from which stability changes can be predicted. In a similar way, one could translate the somewhat more intricate stability exchange theory at bifurcation points into biological terms, but we shall not present that extension here.

\subsection{The $\left(m_{3}, L k\right)$ distinguished diagram}

The key to predicting stability changes is choosing the appropriate quantities to plot in the bifurcation diagram. We call a diagram in which stability information can be determined by the shape of the branches a distinguished bifurcation diagram. For DNA loops, Maddocks and Hoffman show that one distinguished diagram involves $\alpha$ and $d E / d \alpha$ - the derivative of $E(\alpha)$ with respect to $\alpha$-which we abbreviate as $E_{\alpha}$ following standard practice. In this distinguished $\left(E_{\alpha}, \alpha\right)$ diagram, the change in index as a fold is traversed is shown in Fig. 4: the index decreases by one as a leftward-opening fold is traversed upward, and increases by one as a rightwardopening fold is traversed upward.

One can find a compact expression for $E_{\alpha}$ by differentiating the integral form (3) for $E(\alpha)$ via the chain rule, and then integrating by parts and simplifying using the equilibrium equations. The final result is that $E_{\alpha}$ equals the value of the twist moment $m_{3}$ at the point of ring closure $s=0, L$. Consequently, a distinguished diagram involves $\alpha$ and the value of this twist moment $m_{3}$.

Further, due to the simple relationship (9) between $\alpha$ and $L k$, folds in the $\left(m_{3}, \alpha\right)$ and 
$\left(m_{3}, L k\right)$ diagrams exactly correspond and have the same shape. Thus, the $\left(m_{3}, L k\right)$ diagram is also a distinguished diagram, with the same stability exchange rules as shown in Fig. 4. Indeed, referring back to the example bifurcation diagrams in columns a of Figs. 2 and 3, we can see that stability changes at folds in exactly this way, at the points labeled I, II, or IV. Recall that the stability changes at the bifurcation points in Fig. 2 are not covered by the theory in this article, merely the smooth folds in the top figure marked I and IV. Note in addition that jumps in $L k$ play no special role in terms of stability exchange, as is shown in Figs. 2 and 3, where stability remains the same on either side of the horizontal segments in column a.

In the absence of bifurcation points, folds in the $\left(m_{3}, L k\right)$ diagram will generally appear smooth as in Fig. 4. However, in exceptional cases, such as at a bifurcation point, folds can occur at cusps. Although there is a theory of distinguished diagrams for such nongeneric cusped folds $[10,21]$, it will not be discussed in detail here.

Given the stability predictions from the distinguished $\left(E_{\alpha}, \alpha\right)$ diagram, we now describe two additional distinguished diagrams involving $E$, namely $(E, \alpha)$ and $\left(E, E_{\alpha}\right)$. In the DNA example, these diagrams will be $(E, L k)$ and $\left(E, m_{3}\right)$. As the energy $E$ is a central quantity in the problem, such diagrams may be more physically intuitive. These new distinguished diagrams are shown in columns b and c of Fig. 5. They are derived logically from analysis of the $\left(E_{\alpha}, \alpha\right)$ diagram, shown in column a of Fig. 5, with stability exchange information exactly as shown earlier in Fig. 4. The analysis and results differ based on the sign of $E_{\alpha}$ at the fold, so the figure has been divided into a total of 6 cases.

\subsection{The $(E, L k)$ distinguished diagram}

We first consider the $(E, \alpha)$ diagram shown in column b of Fig. 5. In this diagram, the slope of the curve at any point is $E_{\alpha}$. We will use this fact to demonstrate that:

(a) the $(E, \alpha)$ diagram is cusped at the fold in $\alpha$, with the concavity changing at the fold,

(b) the higher-energy branch is more unstable, i.e., has index one greater than the lowerenergy branch.

We will derive these results for Case I of Fig. 5. The other five cases can be handled analogously.

Let $E^{*}$ be the value of the energy at the point $\left(\alpha^{*}, E_{\alpha}^{*}\right)$ in column a, so that the corresponding point in column b is $\left(\alpha^{*}, E^{*}\right)$. A dotted line through the point $\left(\alpha^{*}, E^{*}\right)$ with slope $E_{\alpha}^{*}$ has been drawn in column b. Since the solid branch in column a has $\alpha<\alpha^{*}$ and $E_{\alpha}>E_{\alpha}^{*}$ (at least locally), its image in column b must have slope greater than $E_{\alpha}^{*}$, and therefore lies below the dotted line (and is tangent to it at $\left(\alpha^{*}, E^{*}\right)$ ). Similarly, the dashed branch in column a has $\alpha<\alpha^{*}$ and $E_{\alpha}<E_{\alpha}^{*}$, so its image in column b lies above the dotted line (and is tangent to it at $\left.\left(\alpha^{*}, E^{*}\right)\right)$. Note that as $\alpha$ approaches $\alpha^{*}$, the slope of the upper branch must increase to $E_{\alpha}^{*}$ (and hence the upper branch is concave up), while the slope of the lower branch must decrease to $E_{\alpha}^{*}$ (and hence the lower branch is concave down).

We note that a cusp in the $(E, \alpha)$ diagram where both branches have the same concavity corresponds to the case of a nongeneric cusp fold in the $\left(E_{\alpha}, \alpha\right)$ diagram. Fig. 5 does not include stability exchange information for this case.

As before, because of the close connection between $\alpha$ and $L k$, stability exchange in the $(E, L k)$ diagram is identical to that in the $(E, \alpha)$ diagram derived here. This is confirmed by 
the fact that the predictions of column b of Fig. 5 match the stability changes in the $(E, L k)$ diagrams in Figs. 2 and 3, where cases I, II, and IV occur. Note that the cusps in this diagram are often difficult to see, requiring the zoom views shown in several of the diagrams. Recall that the stability changes at the bifurcation points in Fig. 2, though they look like cusped folds, are not covered by the theory we present in this article, merely the cusped folds in the top figure marked I and IV.

\subsection{The $\left(E, m_{3}\right)$ distinguished diagram}

Given the distinguished diagrams $(E, \alpha)$ and $\left(E_{\alpha}, \alpha\right)$, we may immediately obtain a third diagram $\left(E, E_{\alpha}\right)$, which we show also contains stability exchange information, as depicted in column c. Consider, for example, Case I. We know from column a that the solid branch has a larger value of $E_{\alpha}$ than the dashed branch, and from column b that $E$ is at a local max, so the diagram must be as shown in column c. Similar analysis can be used to derive the figures in Cases III,IV, and VI.

The treatment of cases II and V, where $E_{\alpha}=0$, requires more care. Since the fold in $\alpha$ is open in the $\left(\alpha, E_{\alpha}\right)$ diagram and cusped in the $(E, \alpha)$ diagram, an asymptotic analysis shows that the $\left(E, E_{\alpha}\right)$ diagram is horizontal at $E_{\alpha}=E_{\alpha}^{*}$. Since $E$ does have a local extremum in Cases II and V, the $\left(E, E_{\alpha}\right)$ diagram must therefore have a point of inflection as shown.

In summary, in the $\left(E, E_{\alpha}\right)$ diagram, stability exchanges occur at local extrema of $E$ where $E_{\alpha} \neq 0$, or at points of inflection on the line $E_{\alpha}=0$. This may be readily seen in column c of Figs. 2 and 3 (recall that for DNA, $E_{\alpha}=m_{3}$ ), where instances of cases I, II, and IV, occur. Local extrema of $E$ at $E_{\alpha}=0$ do not correspond to changes of stability (cf. the upper right of Fig. 3).

We note that cusps in the $\left(E, E_{\alpha}\right)$ diagram correspond to cusps in the $\left(E_{\alpha}, \alpha\right)$ diagram, where again Fig. 5 does not give stability information.

\subsection{Summary of distinguished diagrams}

We conclude by summarizing the central result of this article: the stability exchange information available in three bifurcation diagrams involving $m_{3}, L k$, or $E$ :

\begin{tabular}{|c|l|}
\hline Diagram & Stability Exchange Information \\
\hline$\left(m_{3}, L k\right)$ & $\begin{array}{l}\text { Stability index changes at smooth folds in } L k \\
\text { If the fold opens to the right, the bottom branch has lower index } \\
\text { If the fold opens to the left, the bottom branch has higher index }\end{array}$ \\
\hline$(E, L k)$ & $\begin{array}{l}\text { Stability changes at cusped folds } \\
\text { The branch with lower energy has lower index }\end{array}$ \\
\hline $\begin{array}{l}\text { Stability changes at local extrema in } E \text { where } m_{3} \neq 0 \\
\text { or at inflection points in } E \text { where } m_{3}=0\end{array}$ \\
$\begin{array}{l}\text { Stability exchange is as shown in column c of Fig. } 5, \\
\text { depending on the sign of } m_{3}, \\
\text { and whether } E \text { is a local maximum, minimum, or inflection point }\end{array}$ \\
\hline
\end{tabular}




\section{Discussion}

In this article, we have extended previous theory involving $\left(m_{3}, \alpha\right)$ distinguished diagrams to include the more biologically intuitive quantities $L k$ and $E$. In diagrams involving any two of $m_{3}, E$, and $L k$, stability changes can be read off the diagram following the rules in Fig. 5, where $\alpha$ corresponds to $L k$ and $E_{\alpha}$ corresponds to $m_{3}$.

In previous analysis of the perfect rod, the writhe $W r$ has also been identified as a useful indicator in determining stability. For example, Le Bret [19] argues that $d(L k) / d(W r) \geq 0$ is a necessary condition for stability of equilibria of the perfect rod when self-contact is disregarded; Tobias et al [29] have recently extended this result to include a model of self-contact. Le Bret's result can be derived by the analytic techniques used in the distinguished diagram theory that underpin this paper, combined with the fact that the twist strain $u_{3}(s)$ is constant at equilibria of the perfect rod. However, in the case of an intrinsically curved rod, we are unaware of stability results involving $W r$ (for instance, numerical experiments indicate that $d(L k) / d(W r) \geq 0$ is no longer a necessary condition for stability in intrinsically curved rods). In contrast, the distinguished diagrams $\left(m_{3}, L k\right),(E, L k)$, and $\left(E, m_{3}\right)$ derived here can be applied to both perfect and intrinsically curved DNA.

The $(E, L k)$ diagram is notable because of its relation to continuous link distributions $P(L k)$. One model for a nicked DNA minicircle is an equilibrium for which the twist moment $E_{\alpha}$ is zero. And, the equilibrium is stable among all nearby nicked configurations if it is embedded in a local minimum of a stable branch of the $(E, L k)$ diagram. These stable nicked configurations can in turn yield peaks in $P(L k)$. The correlation between $(E, L k)$ diagrams and Monte Carlo simulations of $P(L k)$ in [15] has been quantitatively explored in [7]. Along these lines, we show a final bifurcation diagram in Fig. 6 for a randomly-generated 900-bp DNA from [7]. In this case, there are two points on stable branches where $E$ is at a local minimum, one at $L k \approx 85.4$ and one at $L k \approx 86.4$ (see column b). Thus, this molecule is a candidate for a multipeaked link distribution $P(L k)$. This molecule is also, therefore, a candidate for a nontrivial experimental topoisomer distribution. Although these experiments typically only detect integer $L k$, the $(E, L k)$ diagram should still be the pertinent bifurcation diagram for understanding them. Further investigation is needed to fully understand the connection between the discrete distribution of the integer $L k$ of cyclized molecules and continuous distributions $P(L k)$ of nicked molecules.

There are several other points of interest in the example of Fig. 6. For example, it exhibits a point with Case-III stability exchange, and the behavior in each column agrees with the theoretical predictions in Fig. 5. In addition, the stable solutions in Fig. 6 now lie on two separated branches, so that the stability data in the diagram could not have been readily guessed without the availability of stability tests such as those presented in this paper.

\section{A The writhe frame and a proof of the connection be- tween $L k$ and $\alpha$}

We will use the following several times: 
Lemma 1. Let $\left(\mathbf{d}_{\mathbf{1}}^{\mathbf{a}}, \mathbf{d}_{\mathbf{2}}^{\mathbf{a}}, \mathbf{d}_{\mathbf{3}}\right)$ and $\left(\mathbf{d}_{\mathbf{1}}^{\mathbf{b}}, \mathbf{d}_{\mathbf{2}}^{\mathbf{b}}, \mathbf{d}_{\mathbf{3}}\right)$ be two framings of $\mathbf{r}$, and let $\Omega(s)$ denote the (clockwise) angle of rotation from the first to the second, i.e.

$$
\begin{aligned}
& \mathbf{d}_{1}^{b}(s)=\cos \Omega(s) \mathbf{d}_{1}^{a}(s)+\sin \Omega(s) \mathbf{d}_{2}^{a}(s), \\
& \mathbf{d}_{2}^{b}(s)=-\sin \Omega(s) \mathbf{d}_{1}^{a}(s)+\cos \Omega(s) \mathbf{d}_{2}^{a}(s) .
\end{aligned}
$$

Then $u_{3}^{b}(s)-u_{3}^{a}(s)=\Omega^{\prime}(s)$.

Proof: We note first the following relationships:

$$
\begin{aligned}
\mathbf{d}_{1}^{a} \cdot \mathbf{d}_{1}^{a} & =1, \\
\mathbf{d}_{2}^{a} \cdot \mathbf{d}_{2}^{a} & =1, \\
\left(\mathbf{d}_{1}^{a}\right)^{\prime} \cdot \mathbf{d}_{1}^{a} & =0 \\
\left(\mathbf{d}_{2}^{a}\right)^{\prime} \cdot \mathbf{d}_{2}^{a} & =0 \\
\mathbf{d}_{1}^{a} \cdot \mathbf{d}_{2}^{a} & =0 \\
\left(\mathbf{d}_{1}^{a}\right)^{\prime} \cdot \mathbf{d}_{2}^{a} & =u_{3}^{a}, \\
\left(\mathbf{d}_{2}^{a}\right)^{\prime} \cdot \mathbf{d}_{1}^{a} & =-u_{3}^{a} .
\end{aligned}
$$

(The third and fourth follow from the first and second by differentiating). Using these facts, we directly compute:

$$
\begin{aligned}
u_{3}^{b} & =\left(\mathbf{d}_{1}^{b}\right)^{\prime} \cdot \mathbf{d}_{2}^{b} \\
& =\left(-\sin (\Omega) \Omega^{\prime} \mathbf{d}_{1}^{a}+\cos (\Omega)\left(\mathbf{d}_{1}^{a}\right)^{\prime}+\cos (\Omega) \Omega^{\prime} \mathbf{d}_{2}^{a}+\sin (\Omega)\left(\mathbf{d}_{2}^{a}\right)^{\prime}\right) \cdot\left(-\sin (\Omega) \mathbf{d}_{1}^{a}+\cos (\Omega) \mathbf{d}_{2}^{a}\right) \\
& =\sin ^{2}(\Omega) \Omega^{\prime}+\cos ^{2}(\Omega) u_{3}^{a}+\cos ^{2}(\Omega) \Omega^{\prime}-\sin ^{2}(\Omega)\left(-u_{3}^{a}\right)=\Omega^{\prime}+u_{3}^{a} .
\end{aligned}
$$

We first construct the natural frame $\left(\mathbf{D}_{\mathbf{1}}, \mathbf{D}_{\mathbf{2}}, \mathbf{d}_{\mathbf{3}}\right)[23,17]$. We determine $\mathbf{D}_{\mathbf{1}}$ by solving the IVP $\mathbf{D}_{\mathbf{1}}(0)=\mathbf{d}_{\mathbf{1}}(0), \mathbf{D}_{\mathbf{1}}^{\prime}=-\left\{\mathbf{d}_{\mathbf{3}}\left[\mathbf{d}_{\mathbf{3}}^{\prime}\right]^{T}\right\} \mathbf{D}_{\mathbf{1}}$. With $\mathbf{D}_{\mathbf{2}}$ determined by orthonormality $\left(\mathbf{D}_{\mathbf{2}}=\mathbf{d}_{\mathbf{3}} \times \mathbf{D}_{\mathbf{1}}\right)$, this construction ensures that the twist strain $U_{3}(s)$ of the natural frame equals zero for all $s$ (i.e., the frame is "untwisted") [23]

Next define $\phi(s)$ using the integrand from $W r$ :

$$
\phi(s) \equiv-\frac{1}{2} \int_{0}^{s} \int_{0}^{L} \frac{(\mathbf{r}(\tau)-\mathbf{r}(\sigma)) \cdot\left(\mathbf{r}^{\prime}(\tau) \times \mathbf{r}^{\prime}(\sigma)\right)}{|\mathbf{r}(\tau)-\mathbf{r}(\sigma)|^{3}} d \sigma d \tau .
$$

Now define the writhe frame $\left(\mathbf{d}_{\mathbf{1}}^{\mathbf{w}}(s), \mathbf{d}_{\mathbf{2}}^{\mathbf{w}}(s)\right)$ to be a clockwise rotation by $\phi(s)$ of the natural frame $\left(\mathbf{D}_{\mathbf{1}}(s), \mathbf{D}_{\mathbf{2}}(s)\right)$ about $\mathbf{d}_{\mathbf{3}}(s)$. By Lemma 1 , the twist strain $u_{3}^{w}$ of the writhe frame is

$$
u_{3}^{w}=u_{3}^{w}-U_{3}=\phi^{\prime}(s)=-\frac{1}{2} \int_{0}^{L} \frac{(\mathbf{r}(s)-\mathbf{r}(\sigma)) \cdot\left(\mathbf{r}^{\prime}(s) \times \mathbf{r}^{\prime}(\sigma)\right)}{|\mathbf{r}(s)-\mathbf{r}(\sigma)|^{3}} d \sigma .
$$

Lemma 2. The writhe frame is closed, i.e., $\mathbf{d}_{\mathbf{1}}^{\mathbf{w}}(0)=\mathbf{d}_{1}^{\mathbf{w}}(L)$. 
Proof: Let $\left(\mathbf{d}_{\mathbf{1}}^{*}, \mathbf{d}_{\mathbf{2}}^{*}, \mathbf{d}_{\mathbf{3}}\right)$ be any closed framing of $\mathbf{r}$ with $\mathbf{d}_{\mathbf{1}}^{*}(0)=\mathbf{d}_{\mathbf{1}}^{\mathbf{w}}(0)$; denote its strains by $u_{i}^{*}$ and its twist by $T w^{*}$. Let $\theta(s)$ denote the (clockwise) angle between $\mathbf{d}_{\mathbf{1}}^{*}(s)$ and $\mathbf{d}_{\mathbf{1}}^{\mathbf{w}}(s)$; note that $\theta(0)=0$. By Lemma $1, \theta^{\prime}(s)=u_{3}^{*}(s)-u_{3}^{w}(s)$. Integrating from $s=0$ to $s=L$, and applying (10), we find:

$$
\begin{aligned}
\theta(L) & =\int_{0}^{L} \theta^{\prime}(s) d s=\int_{0}^{L}\left(u_{3}^{*}-u_{3}^{w}\right) d s \\
& =2 \pi\left(T w^{*}\right)+\frac{1}{2} \int_{0}^{L} \int_{0}^{L} \frac{(\mathbf{r}(s)-\mathbf{r}(\sigma)) \cdot\left(\mathbf{r}^{\prime}(s) \times \mathbf{r}^{\prime}(\sigma)\right)}{|\mathbf{r}(s)-\mathbf{r}(\sigma)|^{3}} d \sigma d s \\
& =2 \pi\left(T w^{*}\right)+2 \pi W r=2 \pi\left(T w^{*}+W r\right) .
\end{aligned}
$$

Since $\left(\mathbf{d}_{\mathbf{1}}^{*}, \mathbf{d}_{\mathbf{2}}^{*}, \mathbf{d}_{\mathbf{3}}\right)$ is a closed framing, the link $L k^{*}$ of $\mathbf{r}$ with $\mathbf{r}+h \mathbf{d}_{1}^{*}$ is an integer. Further, we know by the Călugăreanu-White-Fuller formula for closed frames that $L k^{*}=T w^{*}+W r$. Thus, $T w^{*}+W r$ is an integer, so $\theta(L)$ is a multiple of $2 \pi$. Therefore, $\mathbf{d}_{\mathbf{1}}^{*}(L)=\mathbf{d}_{\mathbf{1}}^{\mathbf{w}}(L)$, which implies that $\mathbf{d}_{1}^{\mathbf{w}}(L)=\mathbf{d}_{1}^{*}(L)=\mathbf{d}_{1}^{*}(0)=\mathbf{d}_{1}^{\mathbf{w}}(0)$.

Lemma 3. The link $L k^{w}$ of $\mathbf{r}$ with $\mathbf{r}+h \mathbf{d}_{\mathbf{1}}^{\mathbf{w}}$ is zero.

Proof: Applying the definition of $T w^{w}$ and (10),

$$
\begin{aligned}
T w^{w} & =\frac{1}{2 \pi} \int_{0}^{L} u_{3}^{w}(s) d s \\
& =-\frac{1}{4 \pi} \int_{0}^{L} \int_{0}^{L} \frac{(\mathbf{r}(s)-\mathbf{r}(\sigma)) \cdot\left(\mathbf{r}^{\prime}(s) \times \mathbf{r}^{\prime}(\sigma)\right)}{|\mathbf{r}(s)-\mathbf{r}(\sigma)|^{3}} d \sigma d s=-W r .
\end{aligned}
$$

Thus, $L k^{w}=T w^{w}+W r=0$.

Proposition 1. $L k=m+\frac{\alpha_{0}}{2 \pi}$, where $m \in \mathbb{N}$ is the link of $\mathbf{r}$ with $\mathbf{r}+h \cos \left(\alpha_{0} s\right) \mathbf{d}_{1}(s)-$ $h \sin \left(\alpha_{0} s\right) \mathbf{d}_{2}(s)$.

Proof: Define a floor framing $\left(\mathbf{d}_{1}^{f}, \mathbf{d}_{2}^{f}, \mathbf{d}_{3}\right)$ of $\mathbf{r}$ by

$$
\begin{aligned}
& \mathbf{d}_{1}^{f}(s)=\cos \left(\frac{\alpha_{0} s}{L}\right) \mathbf{d}_{1}(s)-\sin \left(\frac{\alpha_{0} s}{L}\right) \mathbf{d}_{2}(s), \\
& \mathbf{d}_{2}^{f}(s)=\sin \left(\frac{\alpha_{0} s}{L}\right) \mathbf{d}_{1}(s)+\cos \left(\frac{\alpha_{0} s}{L}\right) \mathbf{d}_{2}(s) .
\end{aligned}
$$

By definition of $\alpha_{0}$, the floor framing is closed, i.e., $\mathbf{d}_{1}^{f}(L)=\mathbf{d}_{1}^{f}(0)$. Since by design the clockwise angle of rotation from the floor framing to the real framing is $\Omega(s)=\frac{\alpha_{0} s}{L}$, we have $u_{3}(s)-u_{3}^{f}(s)=\frac{\alpha_{0}}{L}$ by Lemma 1 . Therefore,

$$
\begin{aligned}
T w+W r & =\left(T w-T w^{f}\right)+\left(T w^{f}+W r\right) \\
& =\frac{1}{2 \pi} \int_{0}^{L}\left(u_{3}(s)-u_{3}^{f}(s)\right) d s+L k^{f}=\frac{1}{2 \pi} \int_{0}^{L} \frac{\alpha_{0}}{L} d s+L k^{f}=\frac{\alpha_{0}}{2 \pi}+L k^{f} .
\end{aligned}
$$




\section{References}

[1] S. S. Antman. Nonlinear Problems of Elasticity. Springer-Verlag, New York, 1995.

[2] C. J. Benham. An elastic model of the large-scale structure of duplex DNA. Biopolymers, 18:609-623, 1979.

[3] G. Călugăreanu. Sur les classes d'isotopie des noeuds tridimensionnels et leurs invariants. Czechoslovak Mathematical Journal, 11:588-625, 1961.

[4] N.A. Davis, S.S. Majee, and J.D. Kahn. TATA Box DNA deformation with and without the TATA Box binding protein. J. Mol. Biol., 291:249-265, 1999.

[5] M.D. Frank-Kamenetskii and A.V. Vologodskii. Topological aspects of polymer physics: theory and its biophysical applications. Usp. Phys. Nauk., 134:641-673, 1981. English translation in Sov. Phys. Usp. 24 (1981) 679-695.

[6] F. B. Fuller. The writhing number of a space curve. Proc. Natl. Acad. Sci. USA, 68:815$819,1971$.

[7] P. B. Furrer, R. S. Manning, and J. H. Maddocks. DNA rings with multiple energy minima. Biophysical Journal, 79:116-136, 2000.

[8] L. Greenberg, J.H. Maddocks, and K.A. Rogers. The bordered operator and the index of a constrained critical point. Math. Nach., 219:109-124, 2000.

[9] K. A. Hoffman. Stability of Twisted Elastic Rods. PhD thesis, University of Maryland, 1997. (K. A. Hoffman née K. A. Rogers).

[10] K. A. Hoffman and J. H. Maddocks. Stability of elastic rods. in preparation.

[11] G. Iooss and D.D. Joseph. Elementary stability and bifurcation theory. Springer-Verlag, New York, 1980.

[12] T. Ivey and D. Singer. Knot types, homotopies, and stability of elastic rods. Proc. London Math Soc, 79:429-450, 1999.

[13] H. Jacobson and W. H. Stockmayer. Intramolecular reaction in polycondensations. I. The theory of linear systems. J. Chem. Phys., 18:1600-1606, 1950.

[14] J. D. Kahn and D. M. Crothers. Protein-induced bending and DNA cyclization. Proc. Natl. Acad. Sci. USA, 89:6343-6347, 1992.

[15] V. Katritch and A. Vologodskii. The effect of intrinsic curvature on conformational properties of circular DNA. Biophysical Journal, 72:1070-1079, 1997.

[16] H. Koo, J. Drak, J. A. Rice, and D. M. Crothers. Determination of the extent of DNA bending by an adenine-thymine tract. Biochemistry, 29:4227-4234, 1990. 
[17] J. Langer and D. A. Singer. Lagrangian aspects of the Kirchhoff elastic rod. SIAM Review, 38:605-618, 1996.

[18] M. LeBret. Catastrophic variation of twist and writhing of circular DNA with constraint. Biopolymers, 18:1709-1725, 1979.

[19] M. LeBret. Twist and writhing in short circular DNA according to first-order elasticity. Biopolymers, 23:1835-1867, 1984.

[20] S.D. Levene and D.M. Crothers. Ring closure probabilities for DNA fragments by Monte Carlo simulation. J. Mol. Biol., 189:61-72, 1986.

[21] J. H. Maddocks. Stability and folds. Archive for Rational Mechanics and Analysis, 99(4):301-328, 1987.

[22] R. S. Manning and J. H. Maddocks. Symmetry breaking and the twisted elastic ring. Computer Methods in Applied Mechanics and Engineering, 370:313-330, 1999.

[23] R. S. Manning, J. H. Maddocks, and J. D. Kahn. A continuum rod model of sequencedependent DNA structure. J. Chem. Phys., 105:5626-5646, 1996.

[24] R. S. Manning, K. A. Rogers, and J. H. Maddocks. Isoperimetric conjugate points with application to the stability of DNA minicircles. Proc. R. Soc. London A, 454:3047-3074, 1998.

[25] W. K. Olson. Simulating DNA at low resolution. Current Opinion in Structural Biology, 6:242-256, 1996.

[26] W.K. Olson, A.A. Gorin, X. Lu, L.M. Hock, and V.B. Zhurkin. DNA sequence-dependent deformability deduced from protein-DNA crystal complexes. Proc. Nat. Acad. Sci., U.S.A., 95:11163-11168, 1998.

[27] T. Schlick. Modeling superhelical DNA: Recent analytical and dynamical approaches. Current Opinion in Structural Biology, 5:245-262, 1995.

[28] D. Shore and R. L. Baldwin. Energetics of DNA twisting. J. Mol. Biol., 170:957-981, 1983.

[29] I. Tobias, D. Swigon, and B. Coleman. Elastic stability of DNA configurations. I. General theory. Phys. Rev. E, 61:747-758, 2000.

[30] H. F. Weinberger. On the stability of bifurcating solutions. In L. Cesari, R. Kannan, and H. F. Weinberger, editors, Nonlinear Analysis, pages 219-233. Academic Press, 1978.

[31] J. H. White. Self-linking and the Gauss integral in higher dimensions. American Journal of Mathematics, 91:693-728, 1969. 
$C / A=1.8:$
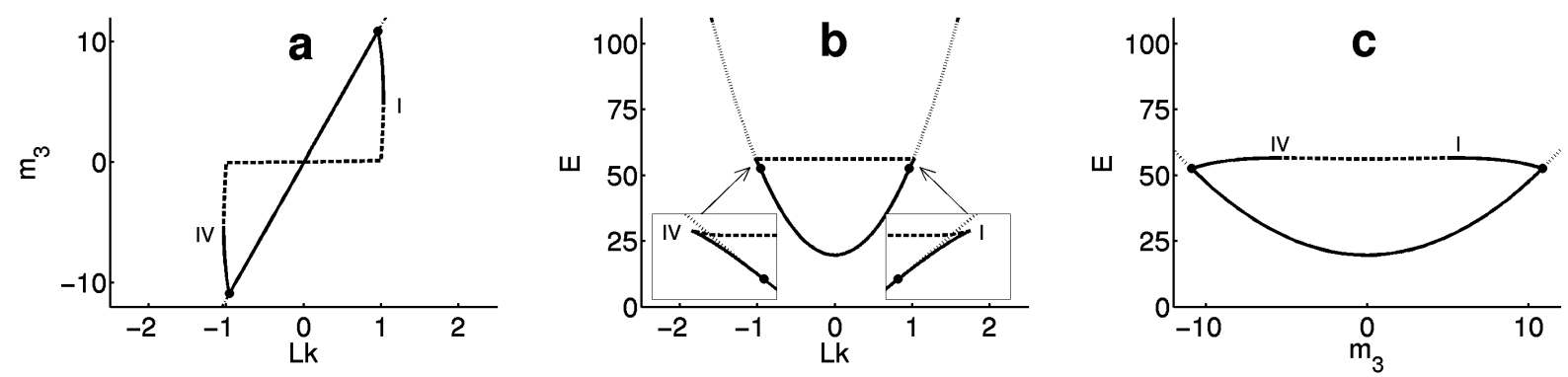

$C / A=1.2:$
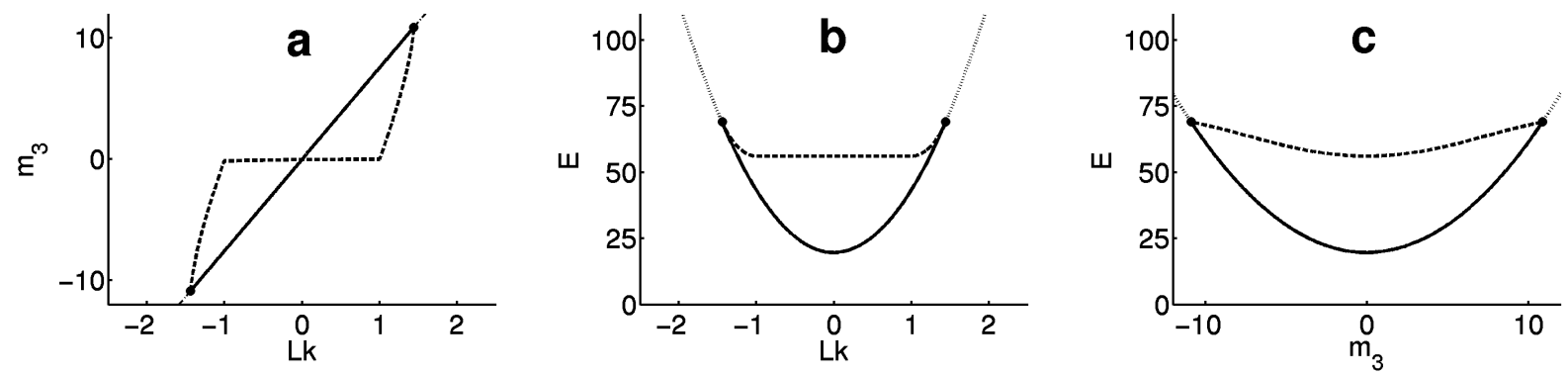

$C / A=0.8$ :
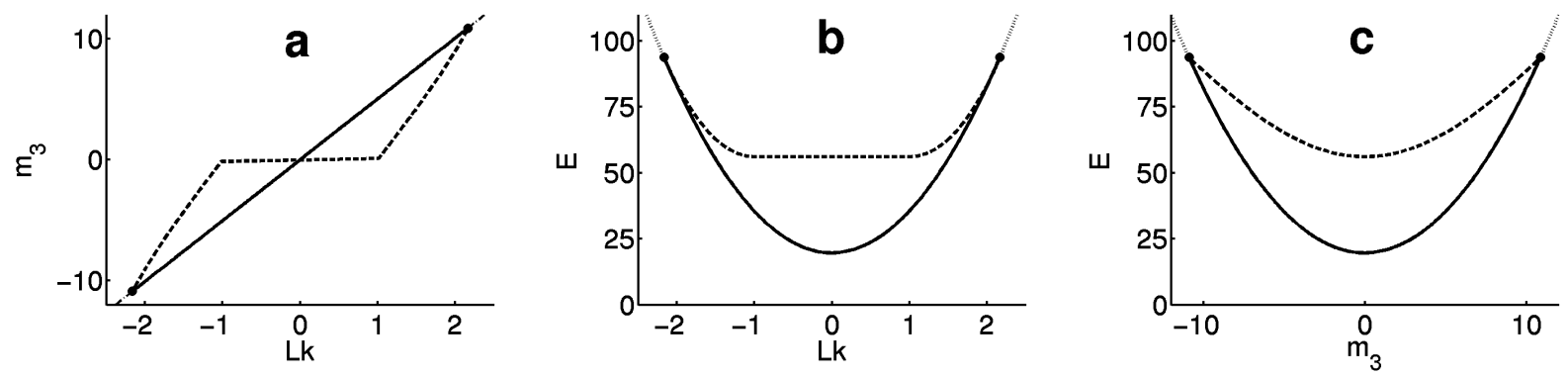

Figure 2: Bifurcation diagrams for an intrinsically straight and untwisted rod with constant and equal bending stiffnesses $K_{1}(s)=K_{2}(s)=A$ and constant twisting stiffness $K_{3}(s)=C$. The link $L k$ is unitless; the quantities $E$ and $m_{3}$ have units of energy (graphed here in units of $A / L)$. Diagrams are shown at three values of $C / A$. Bifurcation points (buckling transitions) are marked by dots. Horizontal line segments in the diagrams in columns a and b occur because $L k$ jumps by 2 at configurations with self-intersection. Stability is indicated by line stylesolid for local minima, dashed for saddles of index one, and dotted for saddles of index two. As discussed in Sec. 5, stability transitions, labeled by I or IV, can be predicted from the shape of the diagram. 
$C / A=1.8$
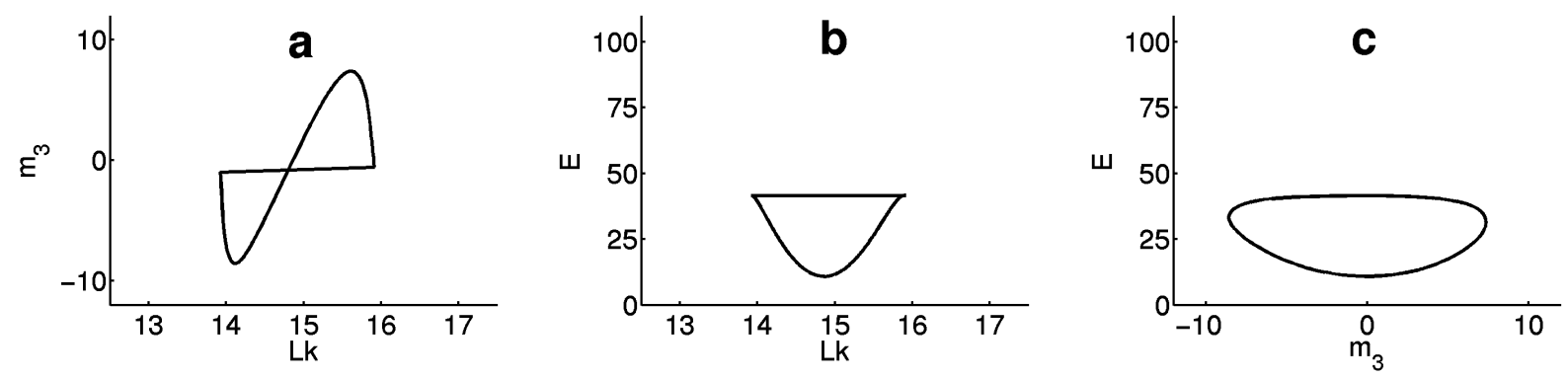

$C / A=1.2:$
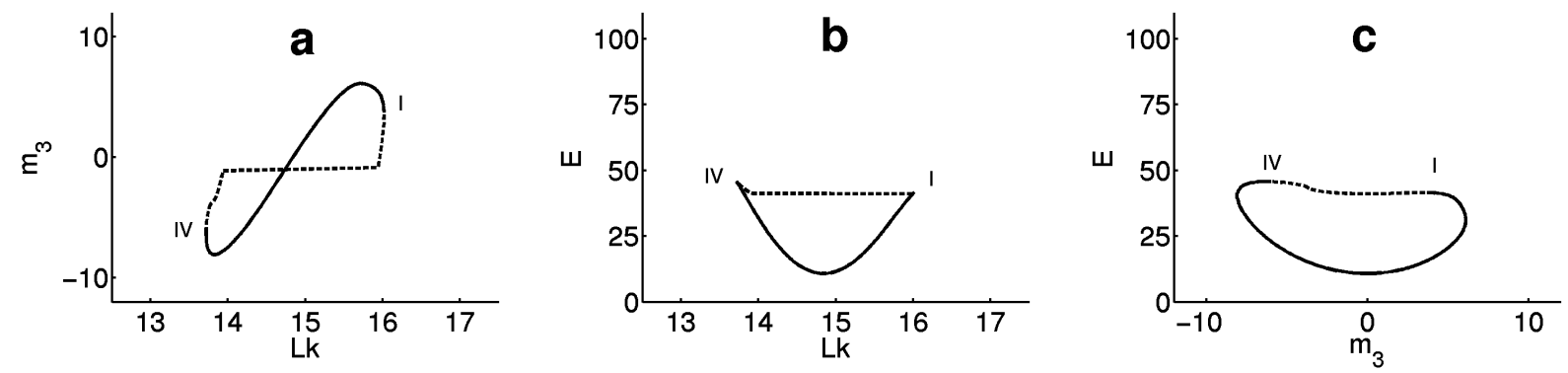

$C / A=0.8$ :
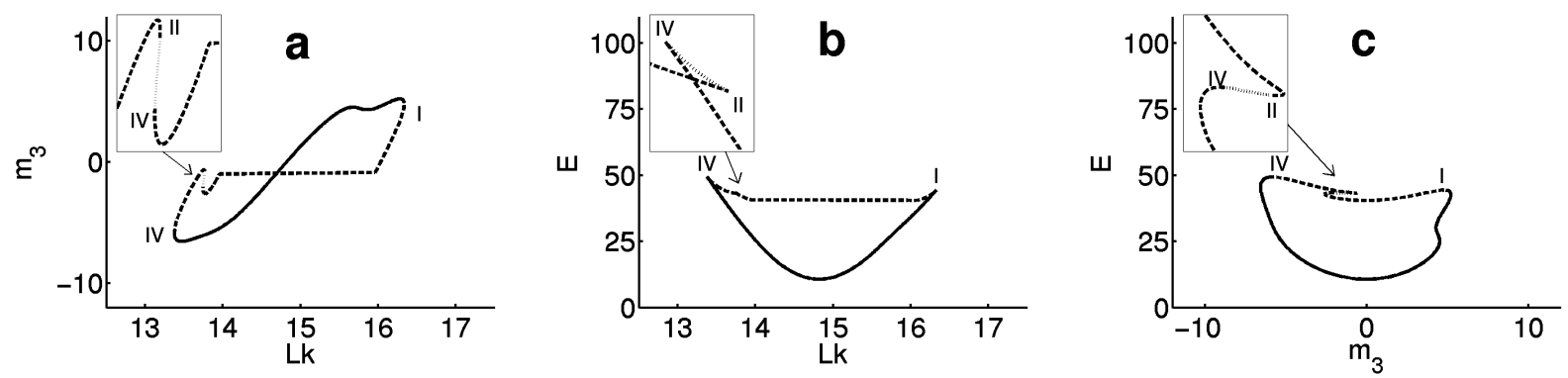

Figure 3: Bifurcation diagrams for an intrinsically curved 155-bp DNA at various values of $C / A$. Because of the symmetry-breaking due to intrinsic curvature, no bifurcation points remain, but stability transitions, labeled I, II, and IV, still occur. Linestyle indicates stability as in Fig. 2. 

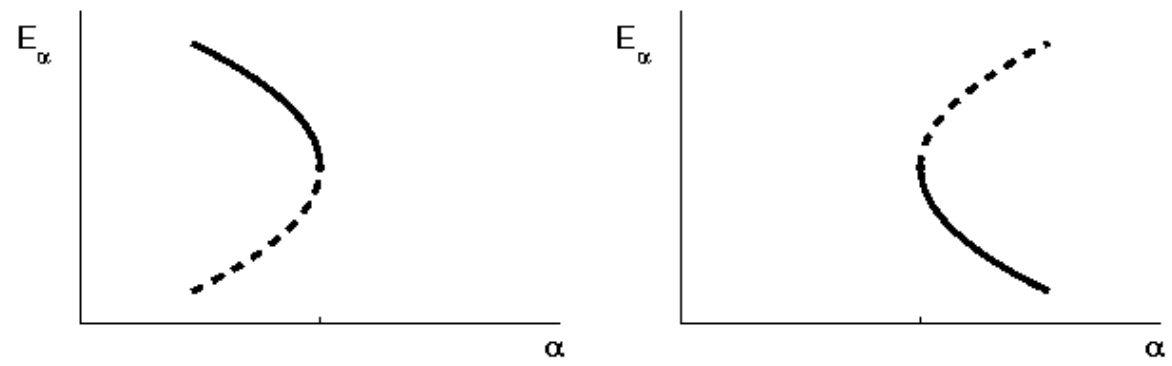

Figure 4: Stability changes at folds in the distinguished bifurcation diagram that plots $E_{\alpha}$ (the derivative of the energy with respect to the angle $\alpha$ ) against $\alpha$. Linestyle denotes the change in index along the branch: the index on the dashed branch is one higher than the index on the solid branch. For example, if the solid branch contains (stable) local minima (index 0), then the dashed branch contains (unstable) saddles with one downward-turning direction (index 1). For the DNA example, $E_{\alpha}=m_{3}$ (at the point of ring closure), and $\alpha$ may be replaced by $L k$, due to (9). 

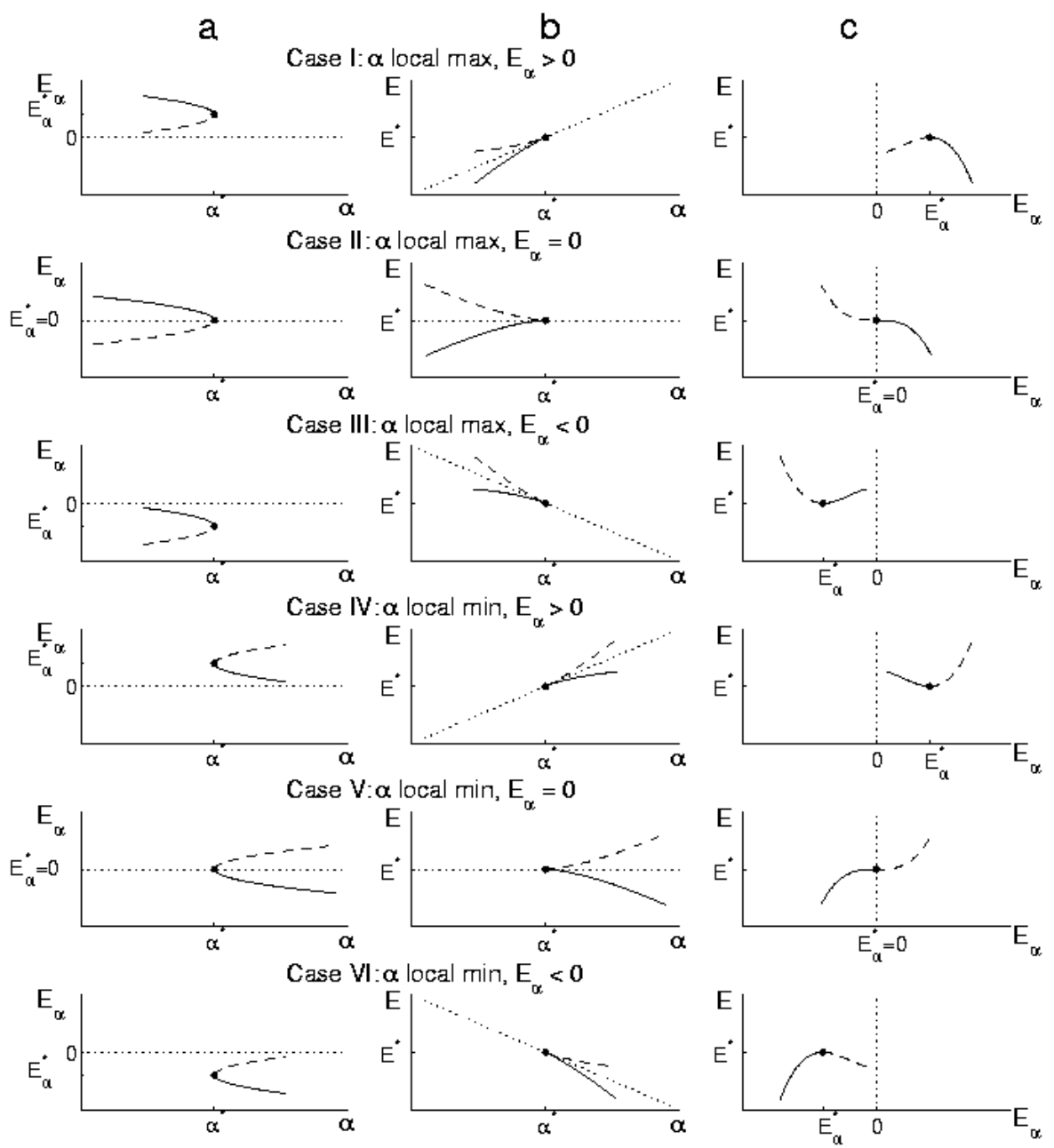

Figure 5: Stability changes at folds in several bifurcation diagrams. For the DNA example, $E$ is the energy, $\alpha$ the twist angle, and $E_{\alpha}$ the twist moment $m_{3}$. Linestyle denotes the change in index along the branch: the index on the dashed branch is one higher than the index on the solid branch. For example, if the solid branch contains (stable) local minima (index 0), then the dashed branch contains (unstable) saddles with one downward-turning direction (index 1). 

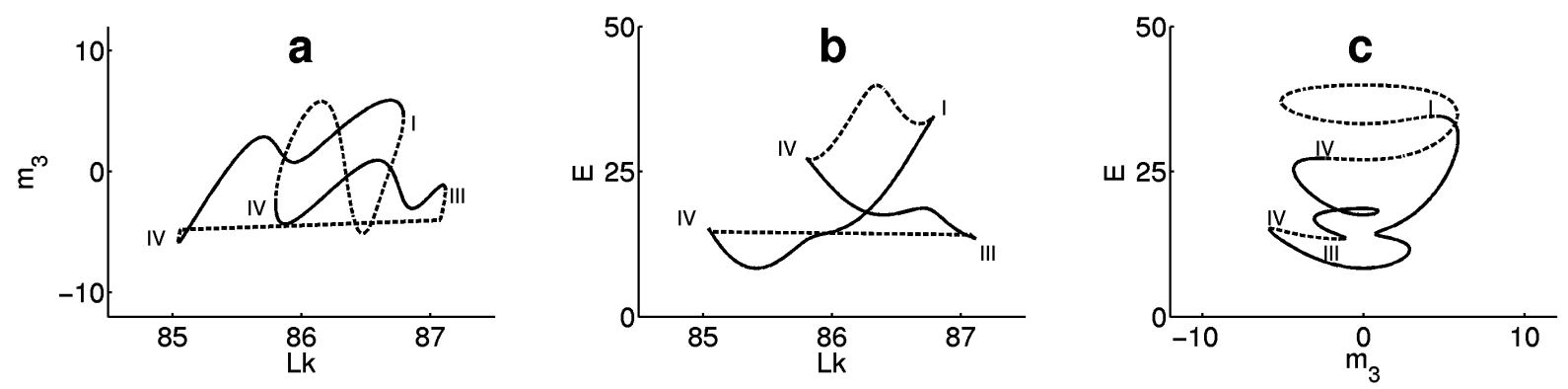

Figure 6: Bifurcation diagrams for a 900-bp DNA (sequence generated at random, $C / A=0.8$ ). Linestyle and labels are as in Fig. 2. 